\title{
Predictive value and utility of oral steroid testing for treatment of COPD in primary care: the COOPT Study
}

This article was published in the following Dove Press journal:

International Journal of COPD

18 November 2009

Number of times this article has been viewed

\author{
Niels H Chavannes ${ }^{1,2}$ \\ Tjard RJ Schermer ${ }^{3}$ \\ Emiel FM Wouters ${ }^{4}$ \\ Reinier P Akkermans ${ }^{3}$ \\ Richard PN Dekhuijzen ${ }^{5}$ \\ Jean WM Muris ${ }^{2}$ \\ Chris van Weel ${ }^{3}$ \\ Onno CP van Schayck ${ }^{2}$ \\ 'Department of Public Health \\ and Primary Care, Leiden \\ University Medical Center, \\ The Netherlands; ${ }^{2}$ Caphri Research \\ Institute, Department of General \\ Practice, Maastricht University, \\ The Netherlands; ${ }^{3}$ Department \\ of Primary and Community Care, \\ Radboud University Nijmegen Medical \\ Center, The Netherlands; ${ }^{4}$ Department \\ of Pulmonary Diseases, University \\ Hospital Maastricht, The Netherlands; \\ ${ }^{5}$ Department of Pulmonary Diseases, \\ Radboud University Nijmegen Medical \\ Center, The Netherlands
}

Correspondence: Niels H Chavannes Department of Public Health and Primary Care, Leiden University Medical Center, PO Box 9600, Zone V0-P, 2300 RC Leiden, The Netherlands

Tel +3I 7I 5268444

Fax $+3|7| 5268259$

Email n.h.chavannes@lumc.nl
Background: The oral prednisolone test is widely used to distinguish chronic obstructive pulmonary disease (COPD) patients who might benefit from inhaled steroid treatment. Previous studies used selected patient groups that did not represent the large COPD population in primary care.

Methods: The study included smokers and exsmokers with chronic bronchitis or COPD from primary care, who underwent prednisolone testing (30 $\mathrm{mg}$ for 14 days) before randomization in a three-year follow-up randomized controlled trial (COOPT Study). Spirometry was performed before and after the test. Responders and nonresponders were classified according to international criteria. Effectiveness of inhaled fluticasone relative to placebo was compared in terms of health status (Chronic Respiratory Disease Questionnaire), exacerbations, and postbronchodilator forced expiratory volume in one second $\left(\mathrm{FEV}_{1}\right)$, using repeated measurement analysis.

Results: Two hundred eighty-six patients recruited from 44 primary care practices were randomized. Nine percent to $16 \%$ of the COPD population was classified as responder, depending on the international guideline criteria used. On average, responders did not reach the minimum clinically important difference in health status ( 0.29 points/year, $P=0.05)$, although a borderline significant effect of inhaled fluticasone was noted. Possible clinically relevant reductions in exacerbation rate (rate ratio 0.67$)$ and $\mathrm{FEV}_{1}$ decline $(39 \mathrm{~mL} /$ year) occurred in responders, but did not reach statistical significance.

Conclusions: Oral steroid testing identifies a limited proportion of COPD patients, but does not reveal any clinically relevant benefit from inhaled steroid treatment on health status. No significant effects on exacerbation rate and lung function decline occurred.

Keywords: COPD, primary care, oral steroid testing, prednisolone test

\section{Background}

In chronic obstructive pulmonary disease (COPD), many different patient groups are represented. Patients include those with chronic bronchitis to the emphysematous, with overweight or with nutritional depletion, and from irreversible obstruction to having a reversible component besides persistent obstruction. By GOLD definition, ${ }^{1}$ and in daily practice, all these groups are termed COPD. In most recent (therapeutic) COPD trials,,$^{2,3}$ however, a strong entry selection occurred to ensure population homogeneity, thereby diminishing external validity. ${ }^{4}$ From large prospective studies ${ }^{2,3,5,6}$ it has become clear that the progressive lung function loss in COPD cannot be altered by inhaled corticosteroid therapy. However, these randomized clinical trials strictly excluded patients with any form of reversibility for methodological reasons. ${ }^{7}$ In practice, the diagnostic prednisolone test has been used widely to identify the patients responding 
to oral steroids, thus foreshadowing the presumable efficacy of inhaled corticosteroid therapy. It is disputed what proportion of COPD patients suffer from persistent obstruction with a reversible component. Estimates differ from 10\% to $30 \%$ depending on clinical setting. ${ }^{7-9}$ It is estimated that $20 \%-30 \%$ of patients with COPD may experience a significant improvement in $\mathrm{FEV}_{1}$ from short-term corticosteroid use. ${ }^{7,10-12}$

In case of persistent obstruction with a significant reversible component, a diagnostic prednisolone test can be performed, although the validity of this test is questioned and different cut-off points for 'response' are advised according to several international guidelines. ${ }^{1,13-15}$ As a consequence, the utility and predictive value of responders within the COPD population is vigorously debated. ${ }^{7,9}$ Small, short-term studies in selected patient groups have described different regimes of prednisolone testing, and as a result the more or less accepted current form (14 days of $30 \mathrm{mg}$ prednisolone) has been part of the diagnostic work-up of COPD-patients. ${ }^{16-19}$ However, the prednisolone test was never validated prospectively in a primary care population. ${ }^{20}$ In severe disease without reversibility it has recently been shown not to be useful by American Thoracic Society (ATS) criteria. ${ }^{21}$ As a result, it was concluded that a short course of oral corticosteroids is a poor predictor of the long-term response to inhaled corticosteroids in COPD. The aim of this study was therefore to determine the predictive value and usefulness of the prednisolone test; to what extent is the clinical efficacy of inhaled corticosteroids (fluticasone propionate $500 \mu \mathrm{g}$ bid) versus placebo related to a positive test response in a primary care population during three years of follow-up.

\section{Methods}

The COOPT trial ${ }^{22}$ is a double-blind, double-dummy, randomized placebo-controlled clinical trial with a three-year follow-up undertaken in The Netherlands between 1998 and 2004. General practitioner (GP)-diagnosed patients with chronic bronchitis and COPD from 44 general practices participated in the study, when postbronchodilator forced expiratory volume in one second $\left(\mathrm{FEV}_{1}\right)$ was between $40 \%-90 \%$ predicted, and $\mathrm{FEV}_{1} /$ forced vital capacity (FVC) was below $88 \%$ (males) or $89 \%$ (females) according to former European Respiratory Society (ERS) criteria. ${ }^{13}$ A clear history of asthma, allergic rhinitis, or atopic eczema was an exclusion criterion, while reversibility to bronchodilators was not. Outcome measures were health status, as measured with the Chronic Respiratory Disease Questionnaire (CRQ), ${ }^{23}$ exacerbation frequency and postbronchodilator $\mathrm{FEV}_{1}$. An exacerbation was defined as an episode with one or more subsequent unscheduled contacts with either a GP or a pulmonologist due to worsening of respiratory symptoms. In this randomized clinical trial a three-leg design was used, with fluticasone propionate and $N$-acetylcysteine as intervention groups, the third leg as placebo. An independent statistician generated a randomization list based on a block size of three for treatment allocation to balance the three treatment arms by study center. Neither investigators nor patients were aware of the group assignment. In this analysis we compared the fluticasone propionate 500 ug twice daily administered as dry powder inhalation by Diskus inhaler versus placebo legs. A wash-out period of three months preceded the study, allowing any effects of stopping inhaled steroids or $\mathrm{N}$-acetylcysteine to subside. During this wash-out phase, $12 \%$ of original study candidates withdrew. In effect, the study group can be described as patients clinically diagnosed as having COPD by their GP, but who did not get worse if inhaled steroids were stopped for three months. Before randomization took place, all patients underwent the prednisolone test, before and after which lung function measurements were taken. The study was approved by the medical ethics review boards of the hospitals involved. All study subjects gave written informed consent.

The diagnostic prednisolone test is generally defined as $\mathrm{FEV}_{1}$ measurements before and after 14 days of $30 \mathrm{mg}$ prednisolone, but cut-off points for a positive response differ among the various international guidelines. The ATS ${ }^{14}$ considered an increase of $>12 \%$ and $200 \mathrm{~mL}$ of baseline $\mathrm{FEV}_{1}$ as a positive response, while the British Thoracic Society (BTS) ${ }^{15}$ stated an increase of $>15 \%$ of baseline $\mathrm{FEV}_{1}$ as a positive response. By contrast, the ERS ${ }^{13}$ used to recommend $\mathrm{a} \geq 10 \%$ increase of $\mathrm{FEV}_{1}$ predicted in their guidelines, but this recommendation has been left out in the 2004 ERS/ATS Guidelines. ${ }^{24}$ Specific criteria for positive response have consequently also been deleted from the British NICE Guidelines ${ }^{25}$ and the executive summary of the GOLD Guidelines. ${ }^{1}$

Differences in longitudinal scores on health status, exacerbation frequency, and postbronchodilator $\mathrm{FEV}_{1}$ were tested on an intention to treat basis, by using statistical techniques for repeated measurements. The placebo group has been taken as representing the natural course, relative to the intervention groups. For statistical testing of differences in exacerbation frequency between placebo and intervention groups during the study period we used a correlated time-event model ${ }^{26}$ (GENMOD-procedure in SAS; Poisson distribution, compound symmetry correlation structure). To analyze the effects on health status, a longitudinal analysis 
was performed on the CRQ-total score, while decline analyses for repeated $\mathrm{FEV}_{1}$ measurements were used for statistical testing of differences in annual lung function. The regression model for these outcomes accounted for correlation between repeated measurements ${ }^{27}$ (PROC MIXED in SAS, compound symmetry correlation structure).

\section{Results}

The study population is described in Table 1 . The number of responders differs from 25 (9\%, ERS) to 44 (16\%, ATS) depending on guideline used. Responders show a significant higher proportion of females, almost equaling men, in comparison with nonresponders across all guidelines. Age, smoking behavior, pack-years, degree of obstruction, and reversibility to bronchodilator do not differ significantly.

The long-term effectiveness of fluticasone propionate relative to placebo in responders versus nonresponders is shown according to different guideline criteria. Outcome variables are exacerbation rate, health status, and postbronchodilator $\mathrm{FEV}_{1}$. On average, clinically relevant differences in health status were not reached. Responders according to ERS experienced a borderline significant effect of inhaled fluticasone on health status $(0.29$ points/year, $P=0.05)$, and to a lesser extent in BTS responders ( 0.26 points/year, $P=0.06)$. ATS-responders did not experience any significant benefit from long-term fluticasone use. Possible clinically relevant reductions in exacerbation rate (rate ratio 0.67 ) and $\mathrm{FEV}_{1}$ decline (39 $\mathrm{mL} /$ year) occurred in BTS responders, but did not reach statistical significance. These effects were similar (rate ratio $0.68, \mathrm{FEV}_{1}$ decline $30 \mathrm{~mL} /$ year) in ERS-responders, while much smaller in ATS responders (rate ratio $0.78, \mathrm{FEV}_{1}$ decline $8 \mathrm{~mL} /$ year).

\section{Discussion}

To our knowledge, this study for the first time prospectively relates the responses to long-term inhaled corticosteroid therapy to different cut-off points for prednisolone testing in primary care. Between $9 \%$ to $16 \%$ of the COPD population was classified as a responder depending on criteria used. On average, clinically relevant differences in health status were not reached. Responders according to the former ERS guideline ( $\mathrm{FEV}_{1}$ increase of $\geq 10 \%$ predicted) experienced a borderline significant effect of inhaled fluticasone on health status $(0.29$ points/year, $P=0.05)$ during three years of treatment. A similar, but not significant effect on health status ( 0.26 points/year, $P=0.06)$ was seen using the former BTS criteria, while ATS responders showed less effect (0.20 points/year). Possible clinically relevant reductions in exacerbation rate (rate ratio 0.67) and $\mathrm{FEV}_{1}$ decline (39 $\mathrm{mL} /$ year) occurred in responders according to BTS, but did not reach statistical significance. Similar results were seen in the ERS group (rate ratio 0.68 and $+30 \mathrm{~mL}$, respectively) but were less similar when the ATS criteria was applied (rate ratio 0.77 and $+8 \mathrm{~mL}$, respectively).

Interestingly, the only other long-term prospective study ${ }^{21}$ that assessed prednisolone testing on these outcomes, but not in a primary care population, concluded on the basis of using ATS criteria that there was 'no relationship between the

Table I Population characteristics of responders versus nonresponders according to former international guidelines ${ }^{13-15}$

\begin{tabular}{|c|c|c|c|c|c|c|c|}
\hline & \multirow[t]{2}{*}{ Total group } & \multicolumn{2}{|l|}{ ERS } & \multicolumn{2}{|l|}{ ATS } & \multicolumn{2}{|l|}{ BTS } \\
\hline & & Responders & Nonresponders & Respodners & Nonresponders & Responders & Nonresponders \\
\hline Number & 286 & 25 & 261 & 44 & 242 & 35 & 251 \\
\hline (\% of total) & & $(9 \%)$ & (91\%) & $(16 \%)$ & $(84 \%)$ & $(12 \%)$ & $(88 \%)$ \\
\hline Age (range) & $59.1(34-76)$ & 59 & 59 & 59 & 59 & 59 & 59 \\
\hline Gender (f/m) & $77 / 209$ & $12 / 13^{*}$ & $65 / 196$ & $18 / 26^{*}$ & $59 / 183$ & $16 / 19 *$ & $61 / 190$ \\
\hline (\% female) & $(27 \%)$ & $(48 \%)$ & $(25 \%)$ & $(4 \mid \%)$ & $(24 \%)$ & $(46 \%)$ & $(24 \%)$ \\
\hline Smoking (curr/form) & $125 / 161$ & $9 / 16$ & $116 / 145$ & $14 / 30$ & III/I3I & $1 \mathrm{I} / 24$ & $114 / 137$ \\
\hline (\% current) & $(44 \%)$ & $(36 \%)$ & $(44 \%)$ & $(32 \%)$ & $(46 \%)$ & $(31 \%)$ & $(45 \%)$ \\
\hline Pack years (SD) & $28.2(17.5)$ & 29 & 28 & 29 & 28 & 31 & 28 \\
\hline FEV , postBD (\%pred) & $69.4(15.6)$ & 69 & 69 & 68 & 70 & 65 & 70 \\
\hline FVC postBD (\%pred) & $89.9(17.7)$ & 94 & 89 & 91 & 90 & 91 & 90 \\
\hline $\mathrm{FEV}_{\mathrm{I}} / \mathrm{FVC}(\mathrm{SD})$ & $62.6(11.8)$ & 60 & 63 & 61 & 63 & 58 & 63 \\
\hline BD-Reversibility (\%) & $6.2(5.1)$ & 6 & 6 & 6 & 6 & 6 & 6 \\
\hline BMI $\left(\mathrm{kg} / \mathrm{m}^{2}\right)$ & $26.3(4.4)$ & 26 & 26 & 25 & 27 & 25 & 27 \\
\hline
\end{tabular}

Notes: *Statistical difference between responders and nonresponders, $P<0.05$.

Abbreviations: ATS, American Thoracic Society; BD, bronchodilators; BMI, body mass index; BTS, British Thoracic Society; ERS, European Respiratory Society; $\mathrm{FEV}_{\text {, }}$, forced expiratory volume in one second; FVC, forced vital capacity; SD, standard deviation. 
short term response to prednisolone and the rate of decline in $\mathrm{FEV}_{1}$ or health status'. In fact, our results are in line with that widely cited study, since we only found any possibly meaningful results using the ERS and BTS criteria. In addition, the Isolde researchers looked at the so-called Callahan criteria ( $\mathrm{FEV}_{1}$ increase of $20 \%$ of baseline), which were derived from a meta-analysis ${ }^{12}$ looking at oral corticosteroid therapy, not prednisolone testing. In fact, this cut-off indeed also showed a significant effect, but this was deemed by the Isolde researchers to be the result of confounding. Thus, the criteria used appears to matter and we cannot recommend the use of the former ATS criteria when performing prednisolone trials, based on our results and on those of the Isolde researchers.

In our study, the proportion of primary care COPD patients meaningfully labeled as responder ranges from 9\% (ERS) to $12 \%$ (BTS), which is considerably lower than expected. We therefore fear that our study was underpowered, since estimates in literature ranged from 20\%-30\% responders. However, it is also possible that we underestimated the number of potential responders in the population, since for ethical reasons our study design provided a wash-out period to exclude all patients that experienced more than two exacerbations when inhaled steroids were taken in the three months preceding entry to the study. ${ }^{28}$ In our population, this means that steroid-dependent patients were not allowed to enter the study.

Contrary to the Isolde researchers, we found a significant gender difference in prednisolone responsiveness across all criteria groups. Women appear particularly prone to COPD when exposed to similar amounts of tobacco ${ }^{29}$ and interestingly, the proportion of female COPD patients responding to prednisolone was consistently higher than among nonresponders at baseline. No other significant differences were seen between responders and nonresponders in terms of age, lung function, current smoking, or pack-years of smoking. This apparent gender difference in prednisolone response therefore deserves further study, since earlier studies probably did not include sufficient numbers of females with COPD, whose prevalence has been seen to rise only in recent years.

In this study, a clear history of asthma, allergic rhinitis or atopic eczema was an exclusion criterion, while reversibility to bronchodilators was not. However, we found no relationship between prednisolone response and bronchodilator reversibility, which was very similar across all criteria groups (Table 1). We consider it highly relevant to clinical practice that reversibility to bronchodilators apparently does not correspond to prednisolone responsibility, since these terms are sometimes interchangingly used as parameters of the same phenomenon. These study data indeed suggest that the response to oral steroids may describe a different underlying inflammatory process than the response to bronchodilation, which is of a fundamentally different origin.

Our results suggest a borderline significant effect on health status in ERS responders, but is it clinically meaningful? The minimum clinical difference on the CRQ is 0.5 points, which is clearly higher than the 0.29 points reached on average in our study, which renders this result marginal. However, the rate ratio for exacerbations $(32 \%$ less than placebo) and even the $\mathrm{FEV}_{1}$ decline $(30 \mathrm{~mL} /$ year less than placebo) point in the same direction in both the ERS

Table 2 Long-term effectiveness of fluticasone propionate versus placebo in responders and nonresponders on exacerbations, health status, and lung function according to different international guidelines ${ }^{13-15}$

\begin{tabular}{|c|c|c|c|c|c|c|c|c|}
\hline \multirow[t]{2}{*}{ Variables } & & \multirow{2}{*}{$\begin{array}{l}\text { Total } \\
\text { group }\end{array}$} & \multicolumn{2}{|l|}{ ERS } & \multicolumn{2}{|l|}{ ATS } & \multicolumn{2}{|l|}{ BTS } \\
\hline & & & Responders & Nonresponders & Responders & Nonresponders & Responders & Nonresponders \\
\hline Exacerbations & FLU & 0.98 & $0.7 I$ & 0.93 & 0.56 & 0.96 & 0.63 & 0.95 \\
\hline (number/yr) & Pla & 0.73 & 1.04 & 0.70 & 0.73 & 0.73 & 0.94 & 0.70 \\
\hline Rate ratio & & 1.30 & 0.68 & 1.33 & 0.77 & 1.32 & 0.67 & 1.36 \\
\hline Health status & FLU & +0.15 & +0.30 & +0.14 & +0.29 & +0.14 & +0.29 & +0.14 \\
\hline (points/yr) & $\mathrm{Pla}$ & +0.13 & +0.01 & +0.13 & +0.09 & +0.13 & +0.03 & +0.13 \\
\hline $\begin{array}{l}\text { Annual difference } \\
\text { (points) }\end{array}$ & & +0.02 & $+0.29 *$ & +0.01 & +0.20 & +0.01 & +0.26 & +0.01 \\
\hline $\mathrm{FEV}_{1}$-change & FLU & -59 & -67 & -58 & -61 & -59 & -60 & -59 \\
\hline$(\mathrm{mL} / \mathrm{yr})$ & Pla & -60 & -97 & -56 & -69 & -58 & -99 & -55 \\
\hline $\begin{array}{l}\text { Annual difference } \\
(\mathrm{mL})\end{array}$ & & +1 & +30 & -2 & +8 & -1 & +39 & -4 \\
\hline
\end{tabular}

Notes: *Statistical difference between responders and nonresponders, $P<0.05$.

Abbreviations: ATS, American Thoracic Society; BTS, British Thoracic Society; ERS, European Respiratory Society; FEV FLU, ; Pla, platelets. 
and BTS groups (33\% less exacerbations and $39 \mathrm{~mL} / \mathrm{year}$ less $\mathrm{FEV}_{1}$ decline, respectively), which may indicate possible clinical relevance. The systematical difference in effects on all three outcomes compared to the nonresponders (and indeed the total group of COPD patients) suggests that this rather small group of responders to prednisolone could be a limited subgroup in primary care, which would need different medical treatment. Since the number of responders (using the ERS or BTS criteria) now can be expected to be around $10 \%$ in a primary care population like ours, we think these results would probably need replication in a larger primary care study. This small but possibly relevant proportion is identical to the $10 \%$ that was found in the earlier meta-analysis on the benefits of oral corticosteroid therapy for COPD patients. ${ }^{12}$ As has been stated before, ${ }^{30}$ it is important to keep an open mind about the possibility that there may be responder and nonresponder subgroups and to continue to seek ways to identify and characterize them, especially in primary care where heterogeneity is common. ${ }^{20}$

\section{Conclusions}

Oral steroid testing distinguishes a limited proportion of COPD patients, but does not reveal clinically relevant benefit from inhaled steroid treatment on health status. No significant effects on exacerbation rate and lung function decline occurred.

\section{Acknowledgments}

The authors would like to thank all participating patients, general practices, and colleagues for their efforts, which enabled the completion of this study. This study was registed at the Dutch Council of Health Insurances (Trial registration number, 98-46140). Funding was provided by the Dutch Council for Health Insurances with complementary funding by the Netherlands Asthma Foundation. Medication was provided by GlaxoSmithKline, Zambon, and Boehringer-Ingelheim. The authors report no conflicts of interest in this work.

\section{References}

1. Rabe KF, Hurd S, Anzueto A, et al; Global Initiative for Chronic Obstructive Lung Disease. Global strategy for the diagnosis, management, and prevention of chronic obstructive pulmonary disease: GOLD executive summary. Am J Respir Crit Care Med. 2007;176(6):532-555.

2. Burge PS, Calverley PM, Jones PW, et al. Randomised double blinded placebo-controlled study of fluticasone propionate in patients with moderate to severe chronic obstructive pulmonary disease: The ISOLDE trial. BMJ. 2000;320:1297-1303.
3. Pauwels RA, Lofdahl CG, Laitinen LA, et al. Long-term treatment with inhaled budesonide in persons with mild chronic obstructive pulmonary disease who continue smoking. European Respiratory Society Study on Chronic Obstructive Pulmonary Disease. $N$ Engl J Med. 1999;340:1948-1953.

4. Herland K, Akselsen JP, Skjonsberg OH, Bjermer L. How representative are clinical study patients with asthma or COPD for a larger "real life" population of patients with obstructive lung disease? Respir Med. 2005;99:11-19.

5. The Lung Health Study Research Group. Effects of inhaled triamcinolone on the decline in pulmonary function in chronic obstructive pulmonary disease. $N$ Engl J Med. 2000;343:1902-1909.

6. Vestbo J, Sorensen T, Lange P, et al. Long-term effect of inhaled budesonide in mild and moderate chronic obstructive pulmonary disease: A randomised controlled trial. Lancet. 1999;353:1819-1823.

7. Calverley PMA. Inhaled corticosteroids are beneficial in chronic obstructive pulmonary disease. Am J Resp Crit Care Med. 2000;161: 341-342.

8. Nisar M, Walshaw M, Earis JE, Pearson MG, Calverley PM. Assessment of reversibility of airways obstruction in patients with chronic obstructive airways disease. Thorax. 1990;45:190-194.

9. Barnes PJ. Inhaled corticosteroids are not beneficial in chronic obstructive pulmonary disease. Am J Resp Crit Care Med. 2000;161:342-344.

10. Chanez P, Vignola AM, O'Shaugnessy T, et al. Corticosteroid reversibility in COPD is related to features of asthma. Am J Resp Crit Care Med. 1997;155:1529-1534.

11. Schayck CP, van Grunsven PM, Dekhuijzen PNR. Do patients with COPD benefit from treatment with inhaled corticosteroids? Eur Respir J. 1996;9:1969-1972.

12. Callahan CM, Dittus RS, Katz BP. Oral corticosteroid therapy for patients with stable chronic obstructive pulmonary disease: a meta-analysis. Ann Intern Med. 1991;114:216-223.

13. Siafakas NM, Vermeire P, Pride NB, et al. Optimal assessment and management of chronic obstructive pulmonary disease (COPD). ERS consensus statement. Eur Respir J. 1995;8:1398-1420.

14. American Thoracic Society. Standards for the diagnosis and care of patients with chronic obstructive pulmonary disease. Am J Respir Crit Care Med. 1995;152:S77-S120.

15. The COPD Guidelines Group of the Standards of Care Committee of the BTS. BTS Guidelines for the management of chronic obstructive pulmonary disease. Thorax. 1997;52(S5):S1-S28.

16. Weir DC, Grove RI, Robertson AS, Burge PS. Corticosteroid trials in non-asthmatic chronic airflow obstruction: a comparison of oral prednison and inhaled beclomethasone dipropionate. Thorax. 1990;45:112-117.

17. Koyama H, Nishimura K, Mio T, Izumi T. Response to oral corticosteroid in patients with chronic obstructive pulmonary disease. Intern Med. 1992;31:1179-1184.

18. Weiner P, Weiner M, Rabner M, Waizman J, Magadle R, Zamir D. The response to inhaled and oral steroids in patients with stable chronic obstructive airway disease. J Intern Med. 1999;245:83-89.

19. Davies L, Nisar M, Pearson MG, Costello RW, Earis JE, Calverley PMA. Oral corticosteroid trials in the management of stable chronic obstructive pulmonary disease. $Q J$ Med. 1999;92:395-400.

20. Chavannes NH, Schermer TRJ; on behalf of the COOPT Study Group. Long-term inhaled steroid response testing should be done in heterogeneous COPD-population. Thorax. 2003;58:648.

21. Burge PS, Calverley PMA, Jones PW, Spencer S, Anderson JA; on behalf of the Isolde Study Group. Prednisolone response in patients with chronic obstructive pulmonary disease: results from the ISOLDE study. Thorax. 2003;58:654-658.

22. Schermer T, Chavannes N, Dekhuijzen R, et al. Fluticasone and $\mathrm{N}$-acetylcysteine in primary care patients with COPD or chronic bronchitis. Respir Med. 2009;103(4):542-551.

23. Guyatt GH, Berman LB, Townsend M, Pugsley SO, Chambers LW. A measure of quality of life for clinical trials in chronic lung disease. Thorax. 1987;42:773-778. 
24. Celli BR, MacNee W, Agusti A, et al. Standards for the diagnosis and treatment of patients with COPD: a summary of the ATS/ERS position paper. Eur Respir J. 2004;23:932-946.

25. National Institute for Clinical Excellence (NICE). Chronic obstructive pulmonary disease. National clinical guideline for management of chronic obstructive pulmonary disease in adults in primary and secondary care. Thorax. 2004;59(Suppl 1):1-232.

26. Diggle PJ, Liang KY, Zeger SL. Analysis of Longitudinal Data. Oxford, UK: Oxford University Press; 1994.

27. Twisk JWR. Applied Longitudinal Data Analysis for Epidemiology. Cambridge, UK: Cambridge University Press; 2003.
28. Schermer TR, Hendriks AJ, Chavannes NH, et al. Probability and determinants of relapse after discontinuation of inhaled corticosteroids in patients with COPD treated in general practice. Prim Care Respir J. 2004; 13:48-55.

29. Prescott E, Bjerg AM, Andersen PK, et al. Gender difference in smoking effects on lung function and risk of hospitalization for COPD: results from a Danish longitudinal population study. Eur Respir J. 1997; 10:822-827.

30. Gross NJ. Responses to steroids and bronchodilators in COPD in the ISOLDE trial: the fat lady sings on. Thorax. 2003;58:647-648.

\section{Publish your work in this journal}

The International Journal of COPD is an international, peer-reviewed journal of therapeutics and pharmacology focusing on concise rapid reporting of clinical studies and reviews in COPD. Special focus is given to the pathophysiological processes underlying the disease, intervention programs, patient focused education, and self management protocols.

\section{Dovepress}

This journal is indexed on PubMed Central, MedLine and CAS. The manuscript management system is completely online and includes a very quick and fair peer-review system, which is all easy to use. Visit $\mathrm{http} / / / \mathrm{www}$.dovepress.com/testimonials.php to read real quotes from published authors.

Submit your manuscript here: http://www.dovepress.com/international-journal-of-copd-journal 